\title{
Procedure for Selecting a Transmission Mode Dependent on the State-of-charge and State-of-health of a Lithium-ion Battery in Wireless Sensor Networks with Energy Harvesting Devices
}

\author{
Vanessa Quintero ${ }^{1}$, Aramis Perez ${ }^{2}$, Francisco Jaramillo ${ }^{3}$, Claudio Estevez ${ }^{4}$, and Marcos Orchard ${ }^{5}$ \\ 1,2,3,4,5 Department of Electrical Engineering, Faculty of Physical and Mathematical Sciences, University of Chile, Santiago, Chile \\ vquintero@ing.uchile.cl \\ aramis.perez@ing.uchile.cl \\ francisco.jaramillo@ing.uchile.cl \\ cestevez@ing.uchile.cl \\ morchard@ing.uchile.cl \\ ${ }^{1}$ Faculty of Electrical Engineering, Universidad Tecnológica de Panamá, Av. Centenario, Panamá, Panamá \\ vanessa.quintero1@utp.ac.pa
}

\begin{abstract}
Diverse methods and considerations have been proposed to manage the available energy in an efficient manner in Wireless Sensor Networks. By incorporating Energy Harvesting Devices in these type of networks it is possible to reduce the dependency of the availability of the Energy Storage Devices, particularly the lithium-ion battery. Recently, the State-of-Charge and State-of-Health of the battery have been considered as inputs for the design of the MediumAccess-Control protocols for Wireless Sensor Networks. In this article, different guidelines are proposed for the design of Medium-Access-Control protocols used in Wireless Sensor Networks with Energy Harvesting Devices considering the State-of-Charge and State-of-Health as indicators for the estimation of the transmission time of the sensor node. The proposed guidelines consider different currents used during the transmission to estimate the State-of-Charge and Stateof-Health of the battery. The incorporation of these indicators aim to improve the decision-making process of the sensor node when transmitting.
\end{abstract}

\section{INTRODUCTION}

Traditionally, Wireless Sensor Networks (WSNs) have used batteries to provide the required energy to the sensor node. Batteries have a limited lifetime, which results in energy efficiency being one of the most studied factors in WSNs. Many of the techniques proposed in the literature to improve energy

\footnotetext{
Vanessa Quintero et al. This is an open-access article distributed under the terms of the Creative Commons Attribution 3.0 United States License, which permits unrestricted use, distribution, and reproduction in any medium, provided the original author and source are credited.
}

efficiency have focused on Medium-Access-Control (MAC) protocols. MAC protocols are designed to conserve energy because they control the communication process of the sensor node, process of a vast energy consumption.

The addition of Energy Harvesting Devices (EHDs) has made possible to have flexibility on the energy requirements, since it has a greater amount of available energy that can be used to power the sensor node or recharge the battery. The EHD has originated a change of guidelines in the design of the MAC protocols to maximize the performance and the network lifetime (Nguyen et al., 2014). MAC protocols for WSN should operate in the Energy Neutral Operation state, i.e., the sensor node has to operate in a condition where the energy consumption is less or equal to the energy harvested. In addition, they need to consider the battery behavior, the energy consumption of the sensor node and the energy available according the type of EHD (Karthi, Rao, \& Pillai, 2015). These four criterion are used to implement dynamic Duty Cycle in MAC protocols for EH-WSN.

In the sensor networks that incorporate EHD, the batteries have an important role in supplying power to the network in the time periods when the EHD cannot do so. Therefore, it is important to understand the behavior of batteries. Two indicators used for this purpose are the State-of-Charge (SOC) and State-of-health (SOH) (Lajara, Solano, \& Pelegri-Sebastia, 2018). The SOC is used to know the percentage of energy available in the battery, while the $\mathrm{SOH}$ indicates the percentage of degradation, i.e., the amount of energy that can be stored and delivered with respect to its nominal value. Both indicators require to be estimated, since they cannot be measured directly, and information from variables such as dis- 
charge current, voltage, temperature, internal impedance, life cycle, capacity degradation rate, is used (Pola et al., 2015). Nowadays, these indicators are being incorporated more frequently in the design of MAC protocols. For example in (Castagnetti, Pegatoquet, Le, \& Auguin, 2014) the authors propose a Duty Cycle control strategy based on the SOC. In (Mokrenko, Vergara-Gallego, Lombardi, Lesecq, \& Albea, 2015), the SOC is related with the remaining battery capacity and the estimation of the SOC is used to apply a Model Predictive Control (MPC). Another example is presented in (Lajara et al., 2018) where the authors proposed a SOH estimation methodology.

The Prognostics and Health Management (PHM) systems use the batterys $\mathrm{SOC}$ and $\mathrm{SOH}$ as indicators regarding the usage and operating decision-making process (Dong, Chen, Wei, $\&$ Ling, 2018). For example, the SOC provides information about the amount of available energy in the battery aiding the decision-making process in the short term to determine if the device has enough energy to execute a specific activity or requires to enter the charging mode. In similar way, is possible to know how degraded a battery is through the $\mathrm{SOH}$. This indicator is used to quantify how much lifetime the battery has. Currently, different methods have been proposed to estimate the battery SOC and SOH (Rezvanizaniani, Liu, Chen, \& Lee, 2014) (Han et al., 2014). The selection of the method will depend on the characteristics of the application and the precision and accuracy required of both indicators. The methods based on battery modeling are used more frequently because they allow on-line estimation. Examples of these methods are Fuzzy Logic, Neural Networks and Bayesian inference (Xu et al., 2014). Regardless of the selected method to estimate the SOC or the $\mathrm{SOH}$, the information provided by these two indicators assists the MAC protocols to adjust their activity or sleep times, thus prioritizing the functions of each of the sensor nodes (Castagnetti, Pegatoquet, Belleudy, \& Auguin, 2012).

This work develops the necessary guidelines for the design of MAC protocols for EH-WSN using the SOC and SOH of a battery. These indicators can improve the decision-making process of the MAC protocols by providing more accurate information of the battery status. According to these indicators, MAC protocols decide the best transmission current, the best periods to charge the battery, and which device has transmission priority. This paper is organized as follows: the first section describes the proposed methodology to develop the guidelines for design of MAC protocols using the SOC and the $\mathrm{SOH}$. The second section details the results obtained by evaluating various scenarios. In the last section we present the conclusions.

\section{METHODOLOGY}

This section describes the methodology used to establish the required guidelines for the design of MAC protocols for EHWSN considering the information provided by the SOC and $\mathrm{SOH}$. The first consideration of the methodology is that each sensor node has a CC2500 transceiver, which has been designed for low-power wireless networks (Instruments, 2013). Table 1 shows some characteristics of this transceiver, specifically the currents used in different operating modes of the sensor node.

Table 1. Currents Characteristics - CC2500

\begin{tabular}{|l||c|}
\hline Operating Mode & Currents \\
\hline Transmission & $22 m A(0 \mathrm{dBm}$ output power $)$ \\
\hline Transmission & $15 m A(-6 \mathrm{dBm}$ output power $)$ \\
\hline Transmission & $11 m A(-12 \mathrm{dBm}$ output power $)$ \\
\hline Reception & $14 m A$ \\
\hline Idle & $1.5 m A$ \\
\hline Sleep & $900 n A$ \\
\hline
\end{tabular}

The second consideration is to define the type of EHD to be used. In this work, it is assumed that each sensor node is provided with a solar panel of $5.5 \times 7 \mathrm{~cm}$. In addition, the periods in which the EHD can harvest energy are determined based on the data of sunlight hours for Santiago of Chile for the year 2017. An average of 10 hours of sunlight is available during each day. Moreover it is considered that during the periods of light the EHD is capable of supplying the necessary current to charge the battery at one-half the nominal current (22.5 $\mathrm{mA}$ for the LIR2032 battery), see Figure 1.

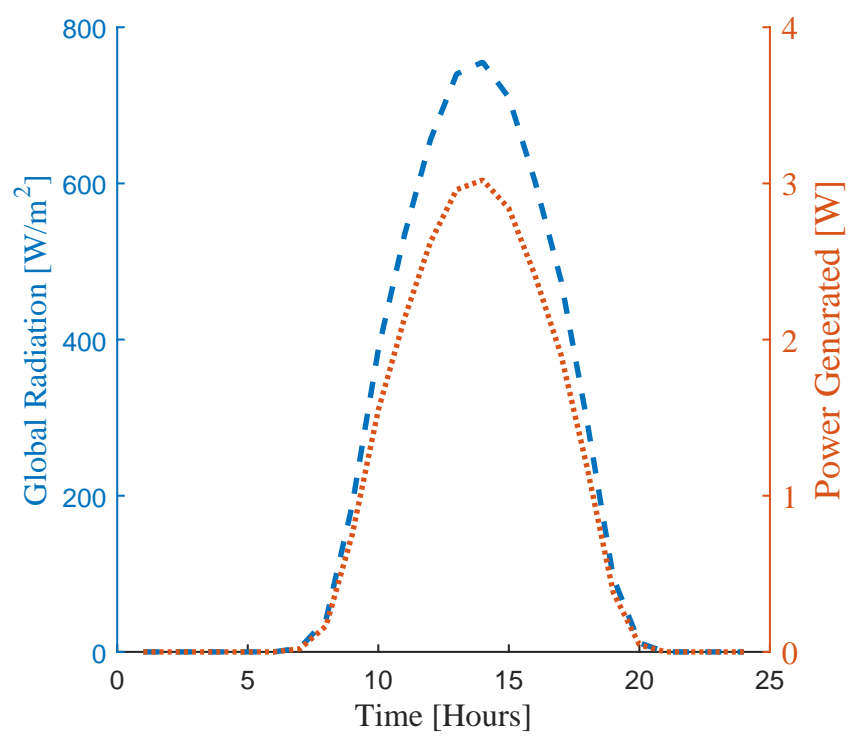

Figure 1. Global Radiation and Power Generated 
The proposed algorithm requires a model that characterizes the battery discharge behavior of a Li-ion battery. In this work, the state-space model presented in (Pola et al., 2015) is used to describe the discharge process. This model defines two states: the first state is an unknown parameter associate to the internal impedance of the battery and the second state corresponds to the SOC estimation. The measurement equation represents the battery voltage at time $k$. The data used to characterize the discharge process was obtained by discharging a Li-ion type LIR2032 $(45 \mathrm{mAh}, 3.6 \mathrm{~V})$ battery at one-half of the nominal current $(0.5 \mathrm{C})$ and constant ambient temperature, as presented in (Quintero, Estevez, \& Orchard, 2017). Figure 2 shows the voltage obtained during the discharge process with its corresponding current profile, which is a combination of two current values. These currents describe two operating modes: transmission mode $(22 \mathrm{~mA})$ and reception mode $(14 m A)$.
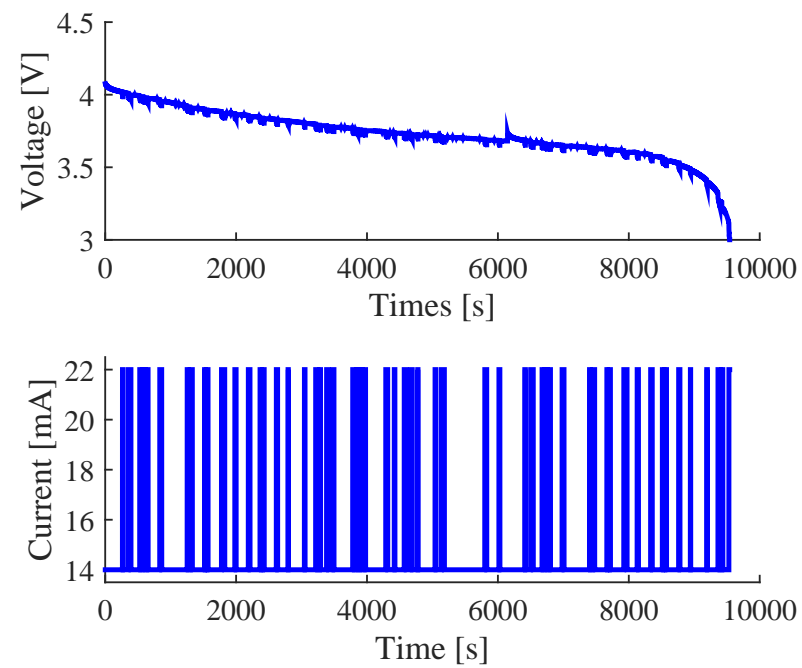

Figure 2. Current and Voltage Discharge

\subsection{SOC Estimation and Prediction}

Initially the SOC estimation is made to establish the point at which the algorithm will begin the prediction of the SOC. In this paper, the SOC estimation and prediction is based on particle filter. Two case studies are presented to evaluate the discharge time of the battery under different current profiles. The first case of study uses an aggressive profile, that is, where the sensor of the node is constantly transmitting, see Figure 3. The second case of study employs a conservative usage profile where the sensor node presents time periods in which no activity is used to evaluate their impact on the discharge process, see Figure 4. This second profile attempts to establish a guideline on how the sensor node should operate at night when it depends exclusively on the energy available in the battery.

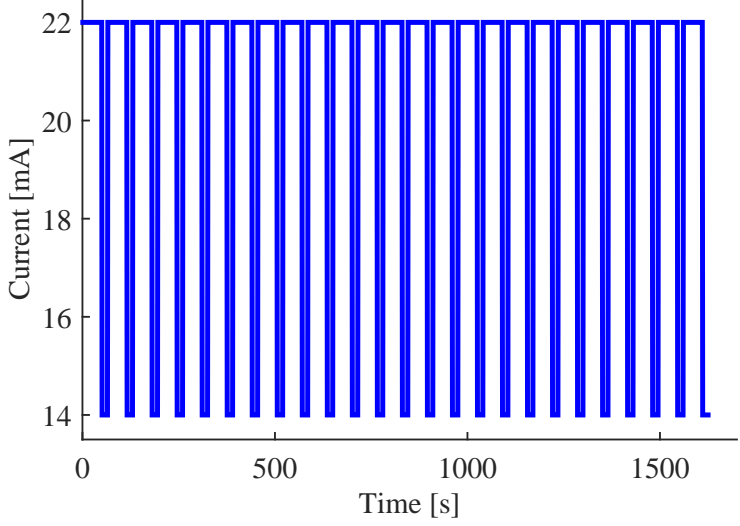

Figure 3. Current Discharge

Subsequently, for each of the proposed scenarios, three transmission currents (11 $m A, 15 m A$, and $22 m A$ ) are used to evaluate their impact on the discharge process. Additionally, the prediction of the SOC starts when the SOC has reached approximately $80 \%$. This condition is established through a random number generator.

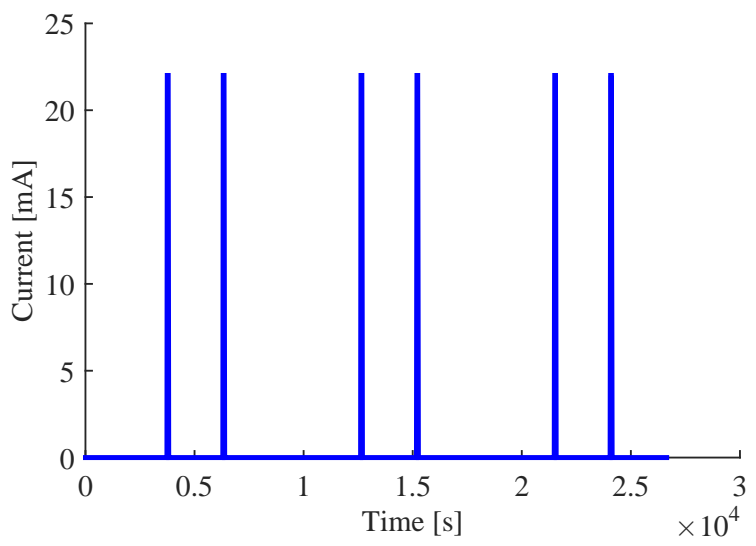

Figure 4. Current Discharge

\subsection{SOH Estimation}

In this subsection, the objective is to analyze the degradation suffered by the battery. To implement this step it is necessary to define an operation cycle. In this work, an operation cycle is fulfilled when the $S O C=0.75$. The conservative current profile is used to evaluate the battery behavior. To evaluate the degradation suffered by the battery, the method proposed in (Pérez et al., 2017) is used. This method uses the concept of Coulombic efficiency to determine the degradation of the battery. Likewise, this method contemplates the use of SOC-swing (SOC-s) and swing range (SR) concepts. The SR indicates the range of start and end of the discharge, while the SOC-s indicates the total variation of the SR. In this case in 
particular the SR is equal to the SOC interval between $75 \%$ and $100 \%$, while the length of the SOC-s is equal to $25 \%$. In addition, the advantages and disadvantages of applying this methods in the design of MAC protocols for EH-WSNs are analyzed.

\section{RESULTS}

The first part of the results obtained corresponds to the estimation and prediction of the SOC of the battery. In the first scenario, the sensor node is constantly transmitting, without resting times. In this case it can be clearly seen that when the transmission current decreases, the transmission time increases by $1 / 3$. This behavior is shown in Figure 5 .

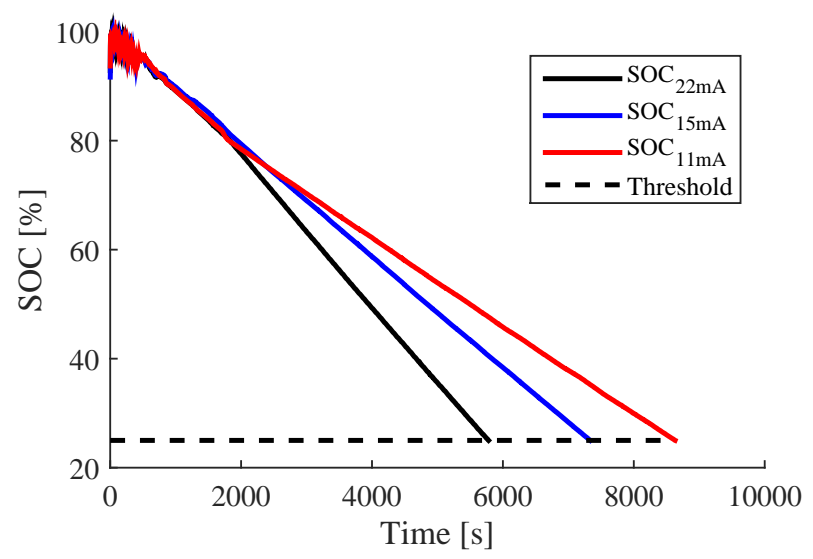

Figure 5. SOC Prediction - Scenario 1

Under the second scenario, the sensor node includes resting times, which conserves energy, emulating a more realistic approach. Figure 6 shows that the SOC does not decay rapidly but remains constant for an instant of time, which coincides with the periods of inactivity of the sensor node. Under this scenario, the typical behavior of the Open Circuit Voltage (OCV) curve of the battery can be observed more clearly, having a $20 \%$ drop at the beginning and then entering the linear area defined by the $0.25 \leq S O C \leq 0.70$.

Through the analysis of the results obtained in Figures 5 and 6 , it is possible to establish the first guidelines to be considered in the design of the MAC protocols for EH-WSNs. This guideline is focused on the way in which the sensor node should transmit at night time. For aggressive profiles it is advisable to transmit to the minimum current allowed by the transceiver, while for profiles, such as the one in Figure 4, operating ranges can be established. Table 2 shows one example of different operating currents according to the SOC of the battery, when a cycle is defined using a SR of $(100 \%$ $25 \%$ ).

The ranges of values shown in Table 2 are reflected in Figure 7, where the use of combined currents for transmission

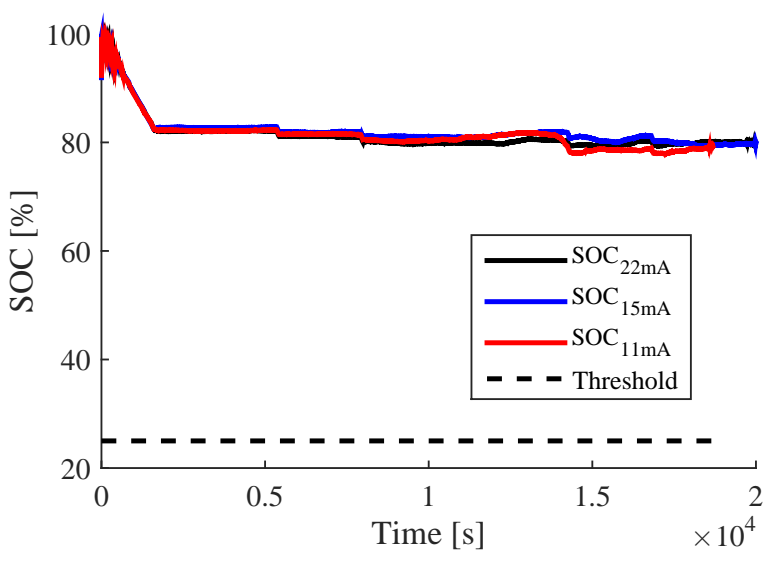

Figure 6. SOC Prediction - Scenario 2

Table 2. SOC operating Ranges

\begin{tabular}{|l||c|}
\hline SOC Ranges & Currents \\
\hline$S O C \geq 0.65$ & $22 \mathrm{~mA}$ \\
\hline $0.25 \leq S O C<0.65$ & $11 \mathrm{~mA}$ \\
\hline
\end{tabular}

is exemplified. When executing the algorithm in this way, it is observed that the transmission time is increased, which improve the operation of the sensor node. In other words, switching to a lower current once the SOC reaches $65 \%$, the battery can deliver energy for nearly 30 minutes more, when undergoing an aggressive usage profile.

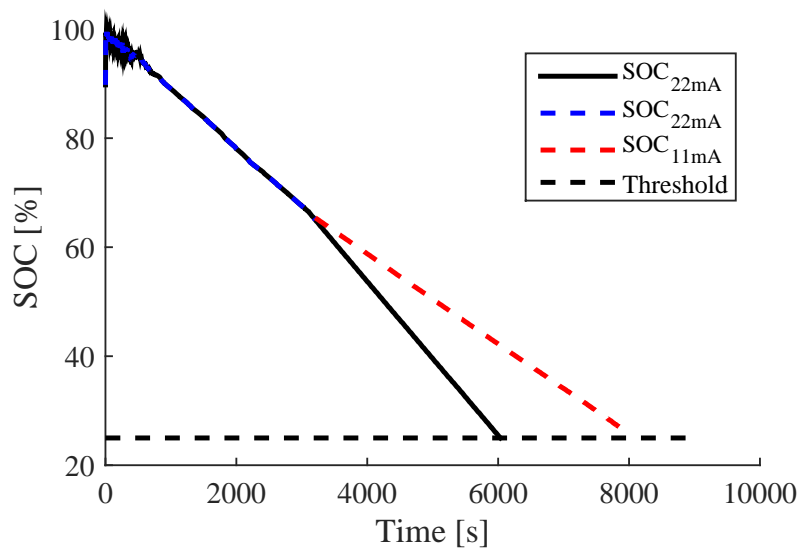

Figure 7. SOC Prediction

To study the degradation suffered by the battery, the algorithm is executed with the conservative current profile during the daylight hours to study the battery charging and discharging processes. Figure 8 shows a portion of time of one of the performed realizations, where the black line represents the $22 \mathrm{~mA}$ current, while the blue and red refer to the cases of $15 \mathrm{~mA}$ and $11 \mathrm{~mA}$, respectively. The main intention of this figure is to illustrate how the battery operates on a daily basis 
for the three discharge currents. In this article, every time the SOC reaches a value of $75 \%$ a cycle is accounted since the algorithm is designed to allow a normal operation of the sensor node above this value. Once the SOC is less that $75 \%$ the battery enters its recharging period, and a new cycle begins. This cycle definition is different from the one described in Table 2, since the SR is different. Figure 9 shows the histogram obtained for the total working time of the sensor node required to complete an operating cycle for the $22 \mathrm{~mA}$ transmission current.

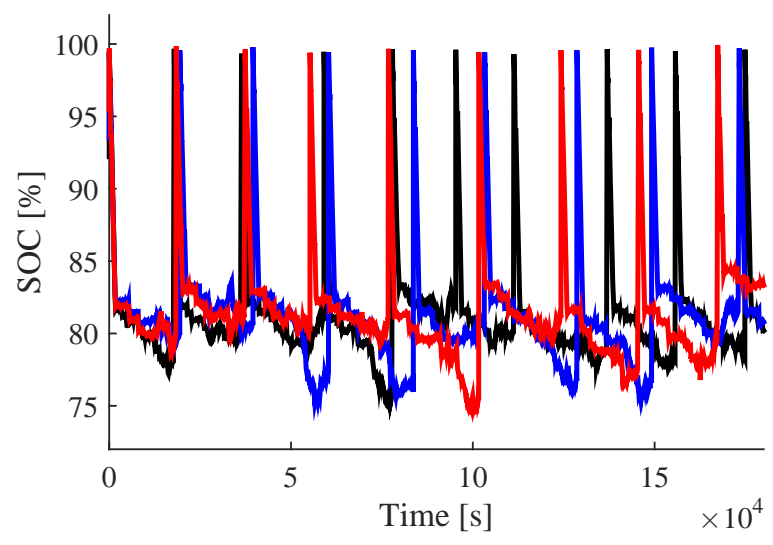

Figure 8. SOC Prediction

Using the information from the datasheet of the LIR2032 battery, the number of the operating cycles and the percentage of degradation suffered by the battery were obtained. The methodology proposed in (Pérez et al., 2017) is used to determine the Coulombic efficiency using a SR (100-75\%), being this $\eta_{k}=0.9995752629$. Using the Coulombic efficiency it is determined that after 25 operating cycles the capacity of the battery has degraded in $0.0017 \%$. The methodology proposed in (Pérez et al., 2017) allows to determine an approximate percentage of degradation suffered by the battery but it does not manage to distinguish when discharging the battery with currents other than $22 \mathrm{~mA}$. Although an important issue to consider is that during the same amount of simulated time, the total cycles reached by the $11 \mathrm{~mA}$ profile was 23 , meaning that the battery degraded less as expected. Furthermore, we can be aware of the maximum degradation that the battery can suffer, since using the current of $22 \mathrm{~mA}$ is transmitting at maximum power, which would be the worst case scenario in terms of energy efficiency of the sensor node.

The following guidelines obtained from the results improve the design of the MAC protocols for EH-WSN. Depending on the type of EHD available in the sensor node it is important to have knowledge of its periods of use, to determine the number of hours in which the sensor node will depend exclusively on the battery. Upon this knowledge the MAC Proto$\mathrm{col}$, with the assistance of the SOC information, must be able

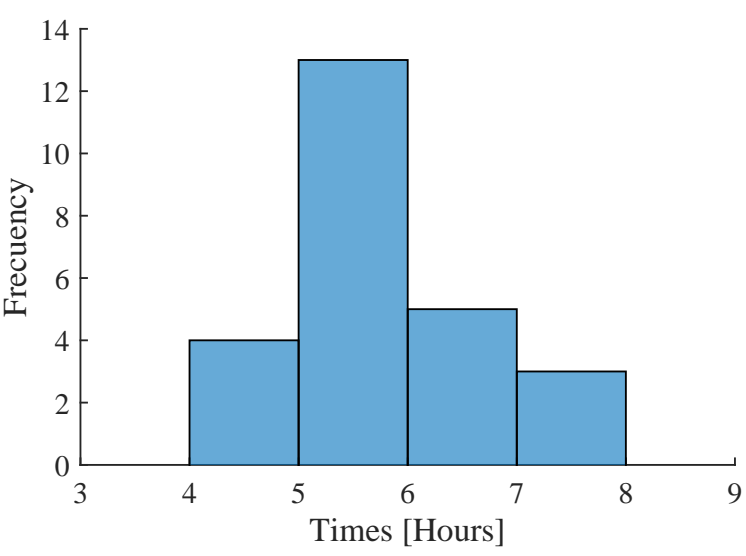

Figure 9. Duration of Each Cycle

to adjust the transmission currents. Incorporating information regarding the battery degradation process aids the sensor node to make timely decisions about the transmission times and about the network lifetime. The design of MAC protocols with SOC and SOH information represents a real challenge for the scientific community because the required tools to estimate both the indicators must be implemented without causing a considerable increase in the energy consumption of the network. On the other hand, it is important to experiment with methods that allow the assessment of the impact on the health status of the battery produced by the combination of different transmission currents.

\section{Conclusion}

Some guidelines were presented to be considered in the design of MAC protocols for EH-WSN that consider SOC and SOH information of the battery. First of all, using different currents in the transmission process helps to increase transmission times and presents the opportunity to select different current combinations to maximize the transmission time when high demand scenarios are present. Second, with inclusion of the $\mathrm{SOH}$ is possible to quantify the degradation process, allowing the search for the best usage profile, that is, the one that causes less degradation in the battery and extends the lifespan of the battery. The incorporation of both estimators entails a great challenge in WSNs, since its implementation has to be done using as less current as possible, although the information provided by both indicators contribute an improvement on the accuracy in the estimation of the transmitting times of each sensor node.

\section{ACKNOWLEDGMENT}

This work has been partially supported by FONDECYT Chile Grant Nr. 1170044, FONDEF ID16I10466, CONICYT PIA ACT1405 and the Advanced Center for Electrical and Electronic Engineering, AC3E, Basal Project FB0008, CON- 
ICYT. The work of Vanessa Quintero was supported by the Universidad Tecnológica de Panamá and IFARHU (Grant for Doctoral Studies) and CONICYT-PCHA/Doctorado Nacional/2016-21161427. The work of Aramis Pérez was supported by the University of Costa Rica (Grant for Doctoral Studies) and CONICYT-PCHA/Doctorado Nacional/201521150121. The work of Francisco Jaramillo was supported by CONICYT-PCHA/Doctorado Nacional/2014-21140201.

\section{NOMENCLATURE}

$\begin{array}{ll}\text { EHD } & \text { Energy Harvesting Devices } \\ \text { EH-WSN } & \text { Wireless Sensor Network with EHD } \\ \text { ESD } & \text { Energy Storage Devices } \\ \text { Li-ion } & \text { Lithium-ion } \\ \text { MPC } & \text { Model Predictive Control } \\ \text { OCV } & \text { Open Circuit Voltage } \\ \text { PHM } & \text { Prognostics and Health Management } \\ \text { SOC } & \text { State-of-Charge } \\ \text { SOH } & \text { State-of-Health } \\ \text { SR } & \text { Swing Range } \\ \text { WSN } & \text { Wireless Sensor Network }\end{array}$

\section{REFERENCES}

Castagnetti, A., Pegatoquet, A., Belleudy, C., \& Auguin, M. (2012). An efficient state of charge prediction model for solar harvesting wsn platforms. In Systems, signals and image processing (iwssip), 2012 19th international conference on (pp. 122-125).

Castagnetti, A., Pegatoquet, A., Le, T. N., \& Auguin, M. (2014). A joint duty-cycle and transmission power management for energy harvesting wsn. IEEE Transactions on Industrial Informatics, 10(2), 928-936.

Dong, G., Chen, Z., Wei, J., \& Ling, Q. (2018). Battery health prognosis using brownian motion modeling and particle filtering. IEEE Transactions on Industrial Electronics.

Han, X., Ouyang, M., Lu, L., Li, J., Zheng, Y., \& Li, Z. (2014). A comparative study of commercial lithium ion battery cycle life in electrical vehicle: Aging mechanism identification. Journal of Power Sources, 251, $38-54$.

Instruments, T. (2013). Cc2500 low-cost low-power $2.4 \mathrm{ghz}$ rf transceiver datasheet.

Karthi, J., Rao, S. V., \& Pillai, S. S. (2015). Duty cycle adapted mac for wireless sensor networks with energy harvesting. In Control communication \& computing india (iccc), 2015 international conference on (pp. 685690).

Lajara, R., Solano, J. J. P., \& Pelegri-Sebastia, J. (2018). Predicting the batteries state of health in wireless sensor networks applications. IEEE Transactions on Industrial Electronics (Early access).
Mokrenko, O., Vergara-Gallego, M.-I., Lombardi, W., Lesecq, S., \& Albea, C. (2015). Wsn power management with battery capacity estimation. In New circuits and systems conference (newcas), 2015 ieee 13th international (pp. 1-4).

Nguyen, K., Nguyen, V.-H., Le, D.-D., Ji, Y., Duong, D. A., \& Yamada, S. (2014). A receiver-initiated mac protocol for energy harvesting sensor networks. In Ubiquitous information technologies and applications (pp. 603-610). Springer.

Pérez, A., Quintero, V., Rozas, H., Jaramillo, F., Moreno, R., \& Orchard, M. (2017). Modelling the degradation process of lithium-ion batteries when operating at erratic state-of-charge swing ranges. In International conference on control, decision and information technologies.

Pola, D. A., Navarrete, H. F., Orchard, M. E., Rabié, R. S., Cerda, M. A., Olivares, B. E., ... Pérez, A. (2015). Particle-filtering-based discharge time prognosis for lithium-ion batteries with a statistical characterization of use profiles. IEEE Transactions on Reliability, 64(2), 710-720.

Quintero, V., Estevez, C., \& Orchard, M. (2017). State-ofcharge estimation to improve energy conservation and extend battery life of wireless sensor network nodes. In Ubiquitous and future networks (icufn), 2017 ninth international conference on (pp. 153-158).

Rezvanizaniani, S. M., Liu, Z., Chen, Y., \& Lee, J. (2014). Review and recent advances in battery health monitoring and prognostics technologies for electric vehicle (ev) safety and mobility. Journal of Power Sources, 256, 110-124.

Xu, J., Mi, C. C., Cao, B., Deng, J., Chen, Z., \& Li, S. (2014). The state of charge estimation of lithium-ion batteries based on a proportional-integral observer. IEEE Transactions on Vehicular Technology, 63(4), 1614-1621.

\section{BIOGRAPHIES}

Vanessa L. Quintero C. received her B.Sc degree from Electronics and Telecommunication Engineering at the Universidad Tecnológica de Panamá (2007). Currently she is a doctorate candidate and a researcher at the Fault Diagnosis and Failure Prognosis Laboratory at the University of Chile, under Dr. Claudio Estevez and Dr. Marcos Orchard supervision. Her research interests include estimation, prognostics with applications to battery and protocols design

Dr. Aramis Perez received his B.Sc. degree and Licentiate degree in Electrical Engineering from the University of Costa Rica. He received his master degree in Business Administration with a General Management Major from the same university. He also received the Doctorate in Electrical Engineering degree from the University of Chile. Currently he is a Research Fellow at the Fault Diagnosis and Failure Prognosis Laboratory and the Energy Center of the University of Chile. Also,he is a Professor at the School of Electrical Engineering at the University of Costa Rica. His research interests 
include parametric/non-parametric modeling, system identification, data analysis, and manufacturing processes.

Francisco Jaramillo received the B.Sc. degree in Electronics Engineering from Universidad de La Frontera, Temuco, Chile, in 2009. Currently he is a doctorate candidate and a researcher at the Fault Diagnosis and Failure Prognosis Laboratory at the University of Chile. His research interests include machine learning, control systems, and estimation and prognosis based on Bayesian algorithms with applications to nitrogen removal in pilot-scale Sequencing Batch Reactors for Wastewater Treatment Plants.

Dr. Claudio Estevez received his B. S. degree in electrical and computer engineering from the University of Puerto Rico, Mayaguez, PR, USA, 2001; his M.S. degree in electrical and computer engineering with a minor in optical engineering from the University of Alabama in Huntsville, Huntsville, AL, USA, 2003; and his Ph.D. degree in electrical and computer engineering and with a minor in computer science from the Georgia Institute of Technology, Atlanta, GA, USA, 2010. In 2011, he was hired as an Assistant Professor by the Electrical Engineering Department at the University of Chile. In 2012, he obtained a [Chilean] national research grant (FONDECYT) to study MAC protocols in WPAN using $60 \mathrm{GHz}$. In the same year he was appointed coordinator of the Communication Networks M.Eng. Program. In 2013, he obtained a [Chilean] national research grant (FONDEF) to study remote polysomnographic monitoring. His research interests include: Connection-oriented transport protocols, network fairness study, MAC-layer protocols in WPAN, 60-GHz WPAN applications, cloud computing with data mining/warehousing, wireless body area networks, remote healthcare.

Dr. Marcos E. Orchard is currently an Associate Professor with the Department of Electrical Engineering, and the head of the Fault Diagnosis and Failure Prognosis Laboratory at the University of Chile, and was part of the Intelligent Control Systems Laboratory at The Georgia Institute of Technology. His current research interest is the design, implementation and testing of real-time frameworks for fault diagnosis and failure prognosis, with applications to battery management systems, mining industry, and finance. His fields of expertise include statistical process monitoring, parametric/nonparametric modeling, and system identification. His research work at the Georgia Institute of Technology was the foundation of novel real-time fault diagnosis and failure prognosis approaches based on particle filtering algorithms. He received his Ph.D. and M.S. degrees from The Georgia Institute of Technology, Atlanta, GA, in 2005 and 2007, respectively. He received his B.S. degree (1999) and a Civil Industrial Engineering degree with Electrical Major (2001) from Catholic University of Chile. Dr. Orchard has published more than 100 papers in his areas of expertise. 